\title{
A Fast Gradient Projection Algorithm for Efficient Cross-Correlation via Sparse Representation in Sensor Networks
}

\author{
Prasant Misra ${ }^{1}$, Mingrui Yang ${ }^{2}$, Wen $\mathrm{Hu}^{2}$, Sanjay $\mathrm{Jha}^{1}$ \\ ${ }^{1}$ School of Computer Science and Engineering, The University of New South Wales, Sydney, Australia. \\ ${ }^{2}$ Autonomous Systems Laboratory, CSIRO ICT Centre, Brisbane, Australia. \\ \{pkmisra, sanjay\}@cse.unsw.edu.au \{Mingrui.Yang, Wen.Hu\}@csiro.au
}

\begin{abstract}
Cross-correlation is a popular signal processing technique used for obtaining reliable range information. Recently, a practical and efficient implementation of cross-correlation (via sparse approximation) was demonstrated on resource constrained wireless sensor network platforms, where the key idea was to compress the received signal samples, and transfer them to central device where the range information was retrieved by $\ell_{1}$-minimization. Although, this mechanism yields accurate ranging results, its applicability is limited due to its slow execution speed and inaccurate recovery of the correlation peak magnitude, which implicitly provides the useful measure of signal-to-noise ratio. In this work, we propose Fast Gradient Projection (F-GP), a new $\ell_{1}$ minimization algorithm, which overcomes the existing limitations, and provides fast and accurate ranging.
\end{abstract}

\section{Categories and Subject Descriptors}

C.3 [Special-Purpose and Application-Based Systems]: Signal processing systems

\section{General Terms}

Algorithms, Design, Experimentation

\section{Keywords}

Cross-Correlation, Sparse Representation, $\ell^{1}$ Minimization, Compressed Sensing, Ranging, Localization

\section{Introduction}

Reliable and robust ranging is an important building block of localization. For high-accuracy range information, acoustic location systems that use a broadband signal design and measure the time-of-flight (TOF) of the acoustic waveform by the mechanism of pulse compression have reported impressive results. However, such systems have not been able to fully optimize their sensing platform (in terms of size, cost and power) to be virtually embedded in the physical world.

Permission to make digital or hard copies of all or part of this work for personal or classroom use is granted without fee provided that copies are not made or distributed for profit or commercial advantage and that copies bear this notice and the full citation on the first page. To copy otherwise, to republish, to post on servers or to redistribute to lists, requires prior specific permission and/or a fee.

SenSys'12, November 6-9, 2012, Toronto, ON, Canada.

Copyright (c) 2012 ACM 978-1-4503-1169-4 ...\$10.00
Although, low-cost and low-power wireless sensor network (WSN) platforms are capable of bridging this gap, they face extreme resource constraints (with limited data sensing rates, link bandwidth, computational speed, battery life and memory capacity) for executing complex algorithms. Recently, a practical and efficient implementation of cross-correlation (via sparse approximation) was demonstrated on resource constrained wireless sensor network platforms [2].

Similar to the design of previous acoustic ranging system, [2] also follows the architecture wherein the transmitter initiates the measurement process by concurrently broadcasting a radio packet to synchronize its local clock with that of the receiver, and a broadband acoustic pulse. The receiver activates its recording circuitry from the time-of-arrival of the radio pulse, acquires the audio samples, and subsequently measures its arrival time. However, its detection and postprocessing method differs significantly in the functionality algorithm on the receiver. Its key idea was to compress the signal samples of the acoustic pulse on the receiver by efficient random projections and transfer them to a base-station (BS), which estimates the range from the limited information by solving the $\ell_{1}$-minimization problem efficiently. The $\ell_{1}$-minimization process does its best to correctly recover the range information by exploiting the signal sparsity in the representation dictionary of the correlation domain.

Although, this mechanism yields accurate ranging results, its applicability is limited for two reasons: (i) slow execution speed, and (ii) inaccurate recovery of the correlation peak magnitude that implicitly results in the loss of signal-to-noise (SNR) information. In this work, we propose Fast Gradient Projection (F-GP), a new $\ell_{1}$-minimization algorithm, that preserves all informations and benefits of the standard crosscorrelation technique, i.e., fast and accurate ranging with implicit measure of SNR.

\section{Fast Gradient Projection $\left(\ell_{1}\right.$-Minimization) Algorithm}

For the mathematical formulation, we adopt the following notation. Let $\mathbf{p} \in \mathbb{R}^{n_{p}}$ and $\mathbf{x} \in \mathbb{R}^{n_{a}}$ represent the transmitted and the received signal vectors, where $0 \leq n_{p}, n_{a} \leq \infty$ and $n_{a} \geq n_{p}$. The detection and post-processing mechanism is implemented in two-phases.

Compression: At the receiver, the dimensions of $\mathbf{x} \in \mathbb{R}^{n_{a}}$ are significantly reduced by multiplying it with a random sens- 


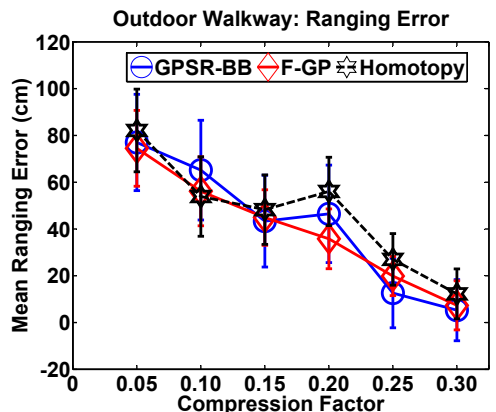

(a)

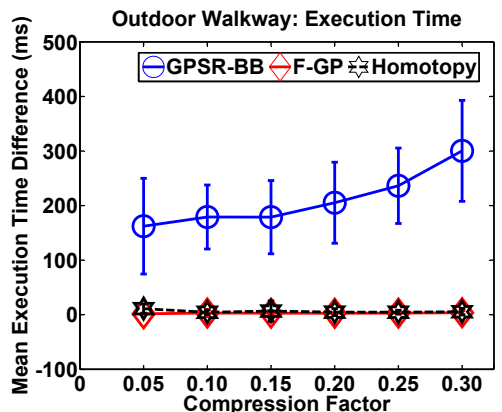

(b)

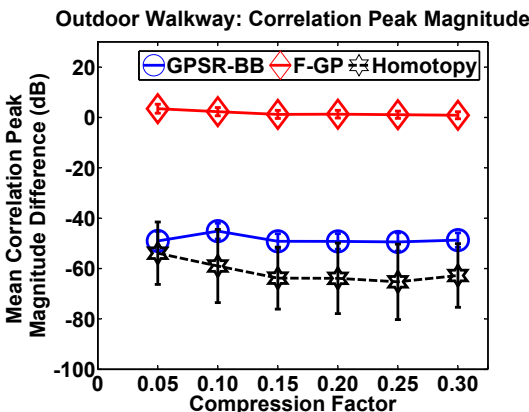

(c)

Figure 1. F-GP vs. GPSR-BB vs. Homotopy: comparison with respect to the (best-case) standard cross-correlation algorithm on the basis of: (i) ranging accuracy, (ii) execution time, and (iii) correlation peak magnitude (SNR).

ing matrix $\Phi \in \mathbb{R}^{m \times n_{a}}$ resulting in the measurement vector $\mathbf{y} \in \mathbb{R}^{m}\left(m \ll n_{a}\right)$ as:

$$
\mathbf{y}=\Phi \mathbf{x}
$$

$m$ is related to $n_{a}$ by the compression factor $\alpha$ given as: $m=\alpha n_{a}$ where $\alpha \in[0,1] . \Phi$ is a binary sensing matrix with its entries identically and independently (i.i.d.) sampled from a symmetric Bernoulli distribution. The $m$ samples of $\mathbf{y}$ are transferred to the BS.

Reconstruction and Detection: The BS requires the apriori knowledge of the seed that generates $\Phi$ and the correlation dictionary $\Psi . \Psi \in \mathbb{R}^{n_{a} \times\left(2 n_{a}-1\right)}$ is the positive and negative time shifted Hankel matrix of p. The importance of $\Psi$ arises from the fact that it provides the representation basis where $\mathbf{x}$ can be sparsely depicted by $\mathbf{s} \in \mathbb{R}^{\left(2 n_{a}-1\right)}$ as:

$$
\mathbf{x}=\Psi \mathbf{s}
$$

The recovery mechanism at the BS reconstructs the sparse correlation coefficient vector $\mathbf{s}$ by solving the following $\ell^{1}$ minimization problem for a given tolerance $\varepsilon$ :

$$
\left(\ell_{r}^{1}\right): \hat{\mathbf{s}}_{1}=\arg \min \|\mathbf{s}\|_{\ell_{1}} \text { s.t: }\left|(\Phi \Psi)^{T} \mathbf{y}-\mathbf{s}\right|_{o} \leq \varepsilon
$$

where $|.|_{o}$ denotes an order metric. The correlation domain coefficients $\hat{\mathbf{s}}_{1}$ are related to the various propagation paths between the transmitter and the receiver, where the index of the first tallest correlation coefficient peak is the estimate of the pulse arrival time of the direct path, and thus, provides the range.

The formulation given by Eq. (3) differs from previous work [1] where, instead of taking the $\|\Phi \Psi \mathbf{s}-\mathbf{y}\|_{2}$ as the performance metric, we aim to make the order difference between the result and the true solution as small as possible. The solution to Eq. (3) is obtained by formulating it as a quadratic problem, and applying a standard gradient projection method to search in the direction of the feasible set. A good choice of the stopping criteria along with other important enhancements make it a fast, practical and efficient method.

\subsection{Empirical Study and Preliminary Results}

We validate the proposed detection method on a proof-ofconcept (POC) acoustic ranging system [2], where the synchronization and ranging signals were generated, captured and analyzed using a laptop. The study was conducted in an outdoor (i.e., very low multipath) environment. The separation distance between the transmitter and the receiver was fixed at $5 \mathrm{~m}$. The transmit power was configured to a level such that 100 observations of the received signal could be recorded within 0 to $5 \mathrm{~dB}$ SNR.

The performance of the F-GP algorithm is compared against two other popular $\ell_{1}$-Minimization algorithms: Gradient Projection for Sparse Reconstruction (GPSR-BB) and Homotopy. Fig. 1 shows the relative mean errors and their deviations with respect to the (best-case) standard crosscorrelation for ranging accuracy, execution time and correlation peak magnitude (indicative of the SNR) accuracy. The results suggest that the proposed F-GP algorithm has the fastest execution speed and the best representation of the correlation peak magnitude among the other two algorithms, which closely approximates the result obtained by the standard cross-correlation technique. However, this improvement comes at the cost of a small $(2 \mathrm{~cm})$ reduction in ranging accuracy compared to the best performing GPSR$\mathrm{BB}$ algorithm.

\section{Conclusion and Future Work}

In this work, we have presented some preliminary results from our project, which has taken an innovative approach to acoustic ranging. Although the results are encouraging, there remains a vast scope for future work and improvement.

- Theoretical analysis and mathematical validation for the fast convergence of the F-GP algorithm.

- Empirical characterization of F-GP in indoor (low and high multipath) environments, where the decrease in signal sparsity could affect its performance.

- Implementation of the acoustic ranging mechanism on commercial-off-the-shelf WSN platforms along with its performance evaluation.

\section{References}

[1] M. A. T. Figueiredo, R. D. Nowak, and S. J. Wright. Gradient projection for sparse reconstruction: Application to compressed sensing and other inverse problems. IEEE Journal of Selected Topics in Signal Processing, 1(4):586-597, 2007.

[2] P. Misra, W. Hu, M. Yang, and S. Jha. Efficient cross-correlation via sparse representation in sensor networks. In IPSN, pages 13-24. ACM, 2012. 